\title{
$\beta-1,6$-linked Galactofuranose-rich Peptidogalactomannan of Fusarium oxysporum is Important in the Activation of Macrophage Mechanisms and as a Potential Diagnostic Antigen
}

\author{
Mariana Ingrid D. S. Xisto ${ }^{1}$, Nathália F. Oliveira. ${ }^{1}$, Gustavo R. C. Santos ${ }^{1}$, Giulia Maria P. Santos ${ }^{1}$, Mariana C.
} Bernardino ${ }^{1}$, Caroline B. Montebianco ${ }^{1}$, Márcio Nucii ${ }^{1}$, Rosa Maria T. Haido², Eliana Barreto-Bergter ${ }^{1}$.

1 Universidade Federal do Rio de Janeiro (UFRJ), Rio de Janeiro, Brazil

2Universidade Federal do Estado do Rio de Janeiro (UNIRIO), Rio de Janeiro, Brazil

Introduction: Fusarium species are common hyaline soil saprophytes and plant pathogens. In humans, they cause superficial infections in immunocompetent individuals, and severe and disseminated diseases in immunocompromised hosts. Recently, two different galactofuranose-containing antigens have been isolated from the cell wall and culture supernatants of Fusarium. As the antigen is released in the culture medium, it could be a target for a Fusarium-specific serological assay. The chemical characterization of fungal antigens is important to allow a rational interpretation of the cross reactivity between pathogenic species, thus helping selection of immunodominant components that can be of diagnostic use. Objectives: Our present study aimed to analyze the major glycoconjugate present on the $F$. oxysporum cell surface by a combination of chemical and spectroscopic methods, and how the glycosylation of $F$. oxysporum glycoconjugate influences the recognition and uptake of $F$. oxysporum. Conclusion: The difference in patient serum reactivity using a PGM from F. oxysporum characterized in the present study as compared with a PGM from $C$. resinae, that presents the same epitopes recognized by serum from patients with aspergillosis, could be considered a potential diagnostic antigen and should be tested with more sera.

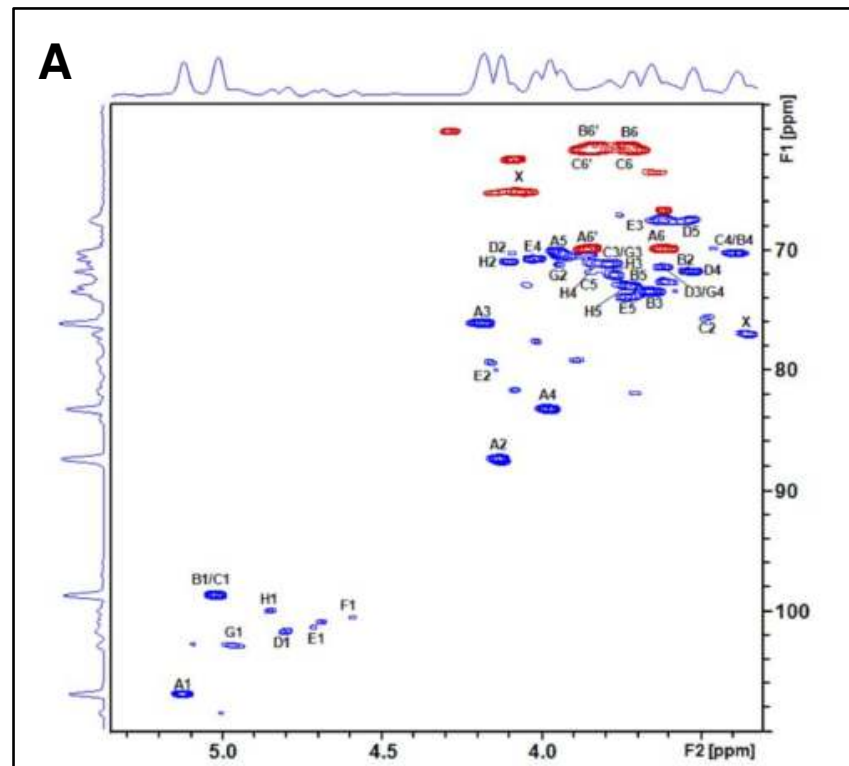

\begin{tabular}{lll}
\multicolumn{1}{c}{ B } & \multicolumn{1}{c}{ Table 1 } & \\
\hline Unit & \multicolumn{1}{c}{ Structure } & \multicolumn{1}{c}{ H1/C1 } \\
\hline A1 & $\rightarrow 2,6)-\beta$-D-Gal $f(1 \rightarrow$ & $5.12 / 106.71$ \\
B1 & $\alpha$-D-Glc $(1 \rightarrow$ & $5.01 / 98.55$ \\
C1 & $\rightarrow 2)-\alpha$-D-Glc $(1 \rightarrow$ & $5.01 / 98.6$ \\
D1 & $\beta$-D-Man $p(1 \rightarrow$ & $4.77 / 101.44$ \\
E1 & $\rightarrow 2)-\beta$-D-Man $p(1 \rightarrow$ & $4.71 / 101.3$ \\
F1 & $\alpha$-D-GlcpA $(1 \rightarrow$ & $4.55 / 100.41$ \\
G1 & $\alpha$-D-Man $p(1 \rightarrow$ & $4.96 / 102.71$ \\
H1 & $\rightarrow$ 6)- $\alpha$-D-Man $p(1 \rightarrow$ & $4.86 / 99.89$ \\
\hline
\end{tabular}

$C$

$\rightarrow 6)-\beta-G a l f(1 \rightarrow 6)-\beta-G a l f(1 \rightarrow$

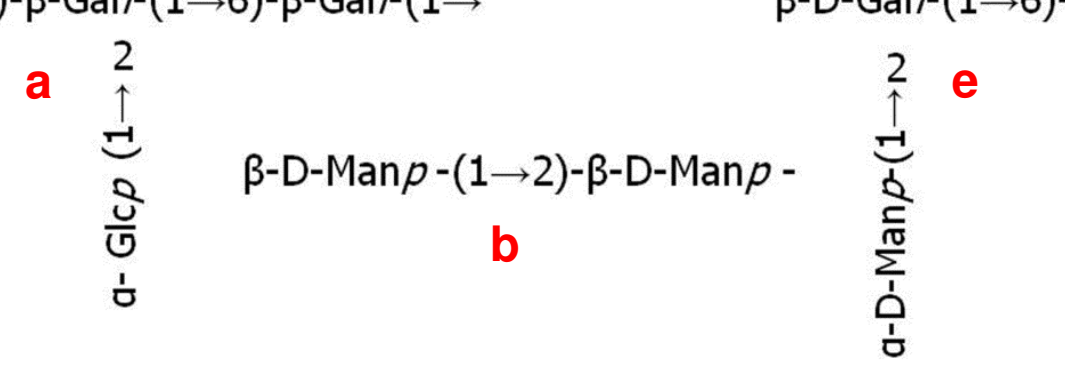

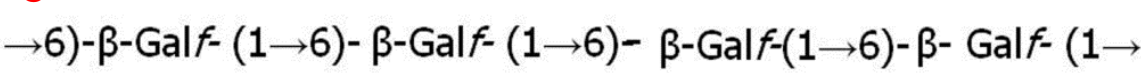

d $\beta$-D-Man $p-(1 \rightarrow 2)-\beta-\mathrm{D}-\operatorname{Man} p-(1 \rightarrow 2)-\mathrm{a}-\mathrm{D}-\mathrm{Gl} c p$

Figure 1: HSQC $\left({ }^{1} \mathrm{H}-{ }^{13} \mathrm{C}\right)$ spectrum of the PGM from F. oxysporum, showing the signals of $\mathrm{CH}$ carbon/proton (phase signals) and those from $\mathrm{CH}_{2}$ (antiphase) (A). The signals and corresponding units were identified in Table 1 (B). Main structures of PGM (C).
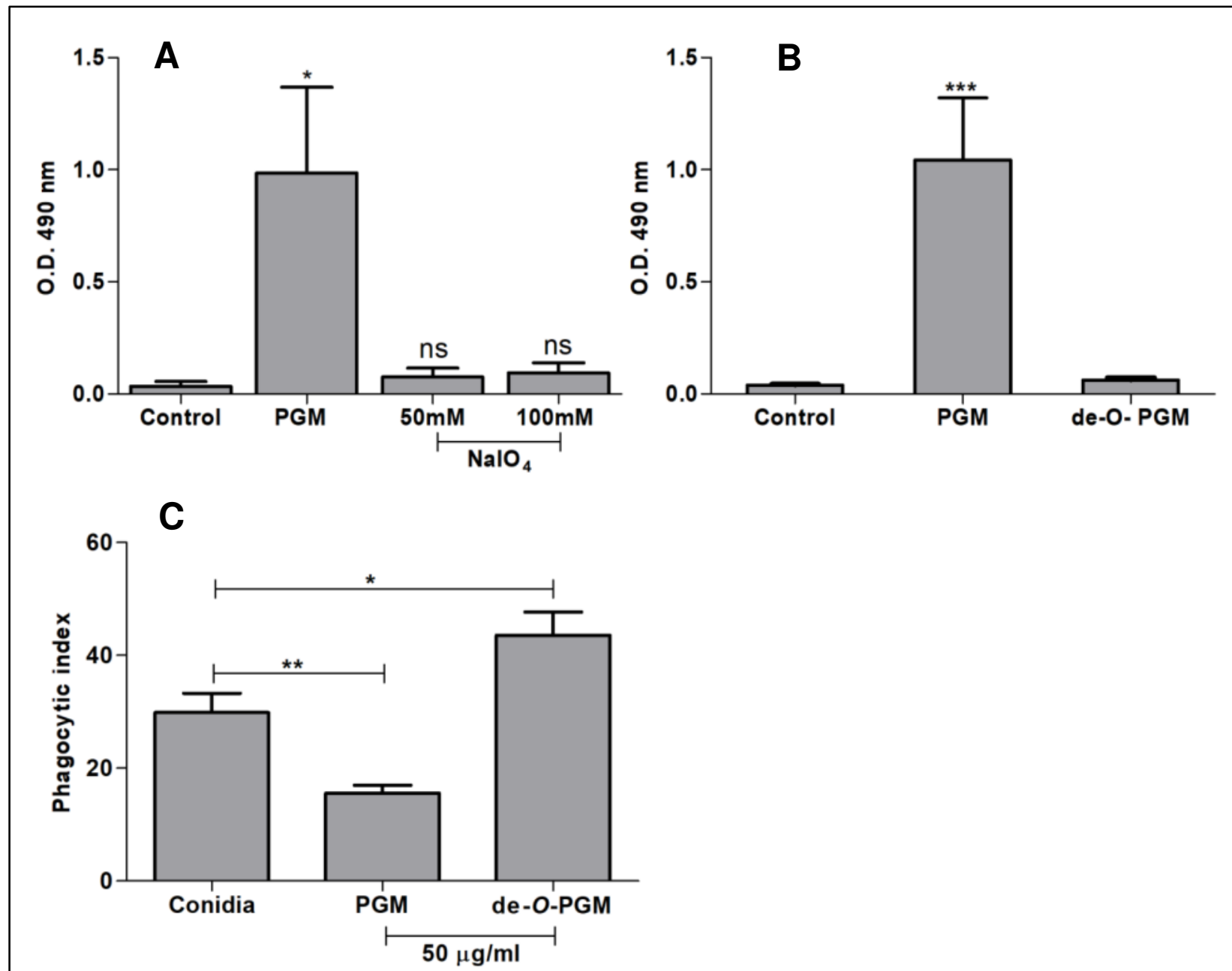

Figure 2: Effect of different treatments in antigenic reactivity of F. oxysporum PGM. (A) Periodate oxidation (50 mM/100 mM) abolished much of the PGM antigenic activity. (B) A strong decrease in reactivity was also observed with de-Oglycosylated PGM. (C) Inhibition assay of the phagocytosis between $F$. oxysporum conidia and mouse peritoneal macrophages by intact and de-O-glycosylated PGM from $F$. oxysporum.

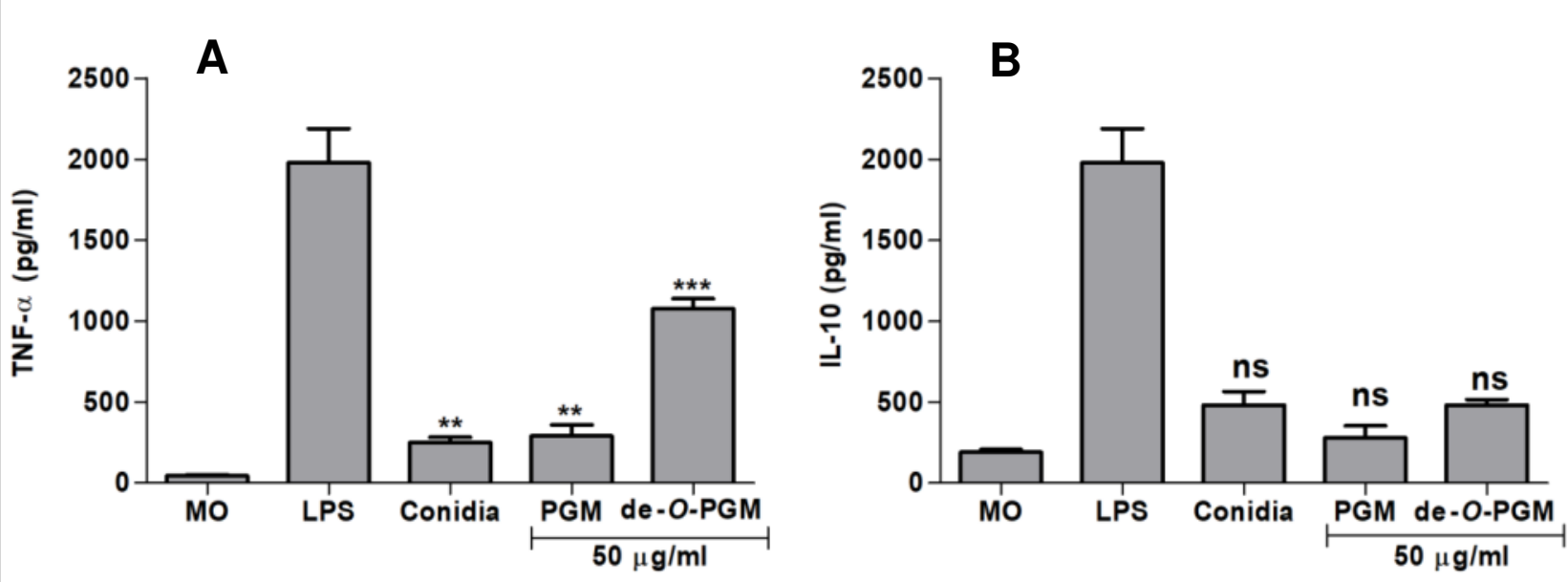

Figure 3: Cytokine release induced by conidia, PGM and PGM de-O-glycosylated from $F$. oxysporum. (A) Both $F$. oxysporum conidia and PGM were able to stimulate the production of TNF- $\alpha$ (A) and IL-10 (B) by macrophages.

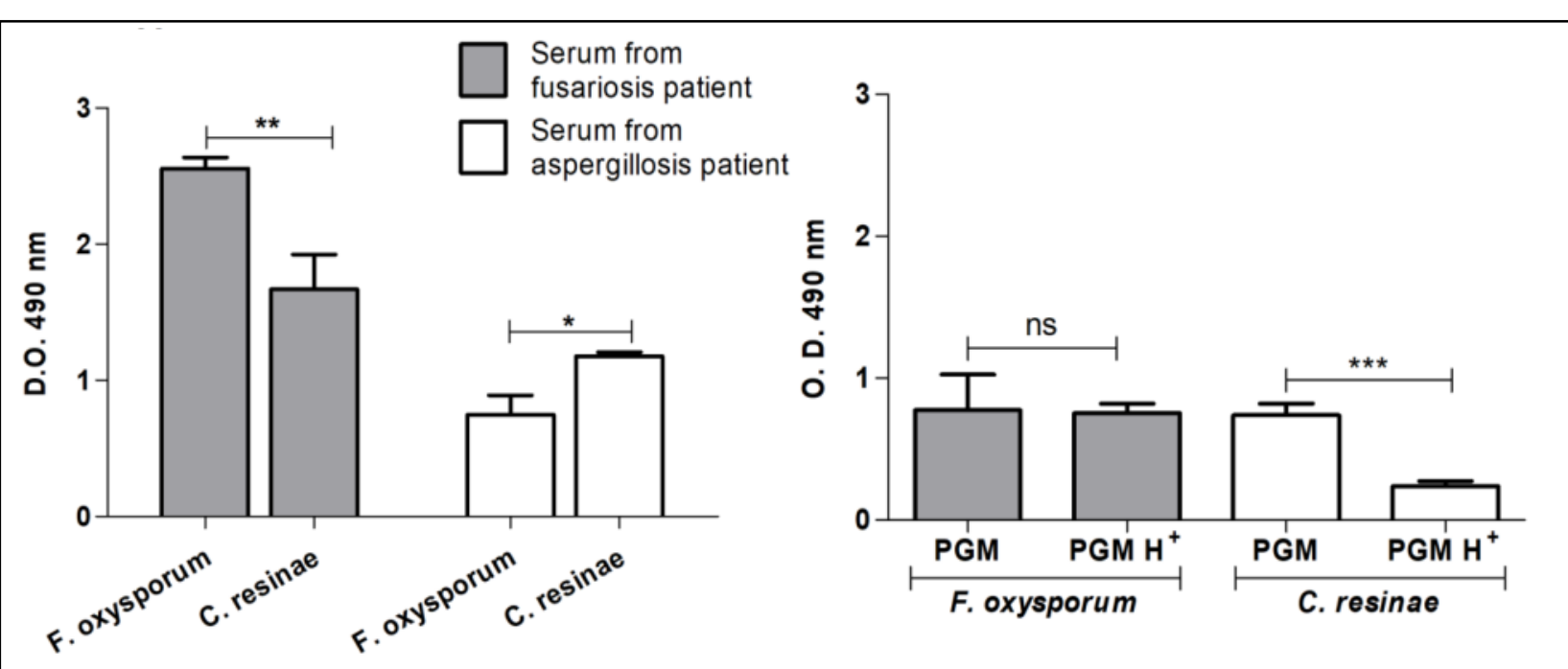

Figure 4: Reactivity of purified PGM from Fusarium and Cladosporium $(\mathbf{A})$ and the effect of Galf $(1 \rightarrow 5)$-side chains removal (B). 